\title{
Design of CMOS Circuit 45mm Technology for Detection of ECG Signal
}

\author{
Gloria Jeanette Rincon Aponte \\ Dept of Computing, University Cooperativa de Colombia, Colombia \\ ingenieriasolidaria@ucc.edu.co
}

Received: $23^{\text {rd }}$ September 2020, Accepted: $3^{\text {rd }}$ December 2020, Published: $31^{\text {st }}$ December 2020

\begin{abstract}
This study ensures that the electrocardiogram (ECG) signals and arythmias are tracked and controlled analogically at the forefront. In line with ECG signal collection schemes, AFE uses methods for medium to minimal architecture and heavy voltage rise to minimize power consumption as well as lower input noise requirements. The AFE has been constructed by a three variable AC-presenting a wide spectrum of biofunctions. AFE is being implemented via $130 \mathrm{~nm}$ CMOS and has a mid-tunable increase of between 31 and $52 \mathrm{~dB}$ with tunable low-pass frequencies and frequencies of high-pass portion. The electricity at $68 \mathrm{nW}$ consumes just $0,5 \mathrm{~V}$ of the input impedance for feedback-reference noise of about $2,8 \mu \mathrm{Vrms}$, as well as a power output factor (POF). The $68 \mathrm{nW}$ AFE low noise was also combined with an ECG bio signals physiological control system. The ECG data from the AFE was tested by monitoring techniques for heart rate detection.
\end{abstract}

Keywords: Electrocardiogram, CMOS, VLSI, Signals

\section{Introduction}

ECG systems are portable, wireless, and economical. ECG data can be collected and sent directly to a clinician for treatment of cardiovascular disease. Easy-to-use ECG gadgets are both convenient and beneficial in the event of an emergency. Many athletes lose their lives as a result of abrupt cardiac arrest in high-intensity sports like soccer and the marathon, for example. A built-in ECG monitoring mechanism in the shirt provides real-time protection. They'll be able to keep track of how the case affects things like ECGs and other ailments. They'll improve their athletic abilities. A heart attack is caused by abnormalities in the electrical system of the heart, which can be identified by the ECG owing to irregular heartbeats.. Sports like soccer, basketball, cycling, and jogging are examples of the kind of events that fall under this category. In biological applications, both signals are of a physical sort. Organic medical signals with millivolt noise at high frequencies. Sensors convert these acoustic signals into electrical signals. The sensor system requires the detection of biological signals, such as EEG, ECG, and EMG, from the human body.

An electrocardiogram (ECG) is the gold standard for detecting heart disease. Single-lead to 12-lead ECG recording devices are available. There are a wide range of ECG acquisition tools in hospitals that allow for highprecision and long-term monitoring. However, patients' ability to move around and take part in activities is hampered as a result. On the other hand, wearable health surveillance devices use multiple sensors to monitor patients in real-time. Selecting sensor types (e.g. wireless or wired), sensor position, number of sensors, and hardware needed for data gathering, storage and transmission are all part of this first stage of an ECG monitoring system's lifespan. Some ECG monitoring systems, on the other hand, handle the acquisition of ECG sensors in real-time and continuously.

For the "human machine" to function effectively, it requires a large number of signals. They are necessary for the coordination and management of the many processes taking place inside our body at any given time. Organs like the heart and lungs generate many of these signals on their own to maintain important activities, such as the 
coordination of the cardiac and respiratory muscles, while the brain generates some of these signals on command. To write the final sentence of this paragraph, these signals travel through nerves to a variety of locations. Signals are transmitted by ionic currents that must be converted to electronic currents in order to be interpreted by electronic instruments. This occurs at the point where the skin meets the electrodes, where the biopotentials are detected. A metallic electrode is placed on the skin and an electrolyte is injected between the electrode and the skin when typical wet electrodes are used. Complex electrochemical processes can take place at this simple and common contact. Valentinuzzi (2004) provides an in-depth look at the skin-electrolyteelectrode interface, but for the purposes of our electronic design, a simplified electrical model will do.

\section{Literature Review}

Village residents and rural healthcare providers are in need of remote wireless ECG self-sufficiency monitoring systems because of inadequate and inequitable infrastructure and skilled medical personnel. As ECG monitoring devices have become increasingly popular in the worldwide healthcare sector, compact, ultra-low-powered, and self-powered versions have emerged. High-performance microcontrollers and radios at cheap prices, advanced sensor technologies, broadband deep drilling, and a market need for wearable biomedical devices have all contributed to an increase in the cost of IC (supply efficient, Devices for non-invasive physiological systems). Exponentially, the price per feature is decreasing. Sensors and actuators that can communicate with each other are being deployed in similar fashion as part of the Internet of Things (IoT). WLANs are also needed to collect and evaluate many biosignals in the daily routine of the users of personal health services (eHealth, mHealth, $u-$ Health, e-Rx, telemedicine, etc.). It's possible to find WBSN systems that are multi-chip prototyped, but they are heavy and consume too much power.

There should be an all-in-one platform that can handle a wide range of biomedical sensor inputs while also being flexible enough to accommodate diverse power harvest modules, signal acquisition/digitalization blocks, local processing units with low capacity, and low-power radios. The size and weight of a battery are constrained by its ability to provide long-term power autonomy. huge battery enhances the soC form factor while small battery requires regular replacements (to make it wearable or disobtrusive). The following methods are used to dissipate power: On-chip data transmitters (iii), lowering the supply voltage (iii), limiting data speeds (iv), and utilising ultra-wide-band radio impulses (v) transmitters all contribute to greater power efficiency. One or more of the following strategies can be used to achieve this decrease.

The analogue block of an IC's supply voltage has less headroom as CMOS technology improves. Digital circuits, on the other hand, benefit from technological scaling by using less power and performing better. Analog components of the SNR, dynamic range, and gain of an IC all suffer from this problem. Dynamic range requirements can have an impact on a signal processing system's power consumption. For example, a system's dynamic range is characterised by its ability to process the largest possible signal without significant distortion to the lowest detectable noise. As a result, new designs that use more digital blocks are needed. A fully digital implementation can't go further until other issues are resolved.

There is a growing interest in biopotential signal acquisition devices including electrooculography (EOG), EEG, ECG, EMG, and axon action potential (AAP) because of advances in CMOS technology, communications, and low-power circuit design techniques (AAP). A biopotential signal can range from tens of millivolts to tens of millivolts in amplitude, as well as from DC to several $\mathrm{kHz}$ in bandwidth. There are a variety of disruptions and artefacts that might affect the ECG signal's characteristics when it is recorded, and this is generally the case. As a result, sifting through the noise to get relevant information is difficult. For example, in the United States, 60 $\mathrm{Hz}$ pickup is common due to inadequate grounding, but in Europe, $50 \mathrm{~Hz}$ pickup is common (in India). It occurs as an impulse or spike at $60 \mathrm{~Hz} / 50 \mathrm{~Hz}$ harmonics, with following spikes occurring at integral multiples of the fundamental frequency of $60 \mathrm{~Hz}$.

Approximately half of the peak-to-peak ECG signal amplitude is represented by its $60 \mathrm{~Hz} / 50 \mathrm{~Hz}$ harmonics. A $60 \mathrm{~Hz}$ notch filter can be used to remove the power line interferences. Sneezing, coughing, or inhaling with significant chest movement may cause baseline drift in ECG measurements taken from the chest. Baseline drift 
can be caused by temperature and bias variations in sensors and amplifiers. Around $0.5 \mathrm{~Hz}$ is the range in which it can be heard. In order to reduce baseline drift, a $0.5 \mathrm{~Hz}$ high pass filter is used.

Electrode skin impedance affects the baseline as the electrode is moved, resulting in motion artefacts. It may give an ECG waveform with greater amplitude. This artefact is 500 percent of the Peak to Peak ECG amplitude and has duration of between 100 and $500 \mathrm{~ms}$. An adaptive filter can be used to reduce motion artefacts. Electrical activity within the muscle is usually what triggers a muscular contraction. It's widely accepted that muscle contraction signals are a band-limited kind of Gaussian noise with a zero-mean mean. Electromyogram (EMG) interference causes a rapid variation in the ECG wave that is significantly faster than the EMG wave. Dc to $10 \mathrm{kHz}$ and a period of 50 milliseconds are the range and frequency of this signal In order to remove EMG interference, a morphological filter of a unit square-wave structure (ideal width is $0.07 \mathrm{~s}$ ) is used.

When the ECG signal does not show any disease or abnormalities, it is said to be in normal sinus rhythm (NSR). NSR's heart rate ranges from 60 to 100 beats per minute. The regularity of the R-R interval varies a little depending on the breath cycle. At a heart rate of more than 100 beats per minute, sinus tachycardia ensues. When blood flow increases, the heart's normal response is to pump more blood through the body.

Definition of Problem: Instrument amplifier noise power trade-offs. One parameter (noise and power dissipation) is emphasised in many of these papers, while the importance of the other factors is minimised. As a result of this need, recent research has focused on low-power and noise ECG systems. Furthermore, a network that can effectively cover the entire frequency range of an ECG signal must be fully integrated. The long-term, fast-moving feature demands large resistors and capacitors. In order to achieve this constant duration with a large resistor, a variety of designs have been proposed, the majority of which need the use of a wide capacitor. Developing an ECG amplifier with reduced noise for better signal reception and low voltage for active monitoring is a difficult challenge.

\section{Research Methodology}

Transistor Level Implementation of operational Transconductance Amplifier - It is usual for OTA to use the OTA mirror topology. Low-power applications can benefit from the new OTA mirror's headroom advantage, as well as the fact that voltage supplies have a minimal impact on the design. The entire differential current mirror of the OTA.

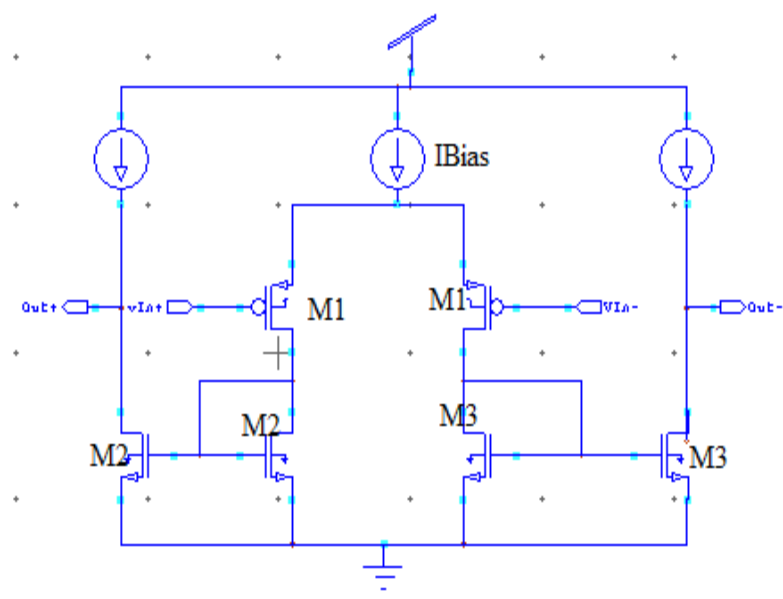

Fig 1: Fully differential current mirror OTA

In order to determine the $\mathrm{Gm}$, the mirror ratio $(\mathrm{K})$ must be known. DC gain rises in direct proportion to monitoring ratio. However, when $\mathrm{K}$ rises, the mirror transistor's gain is proportionate, reducing the parasitic and increasing the bandwidth of the mirror node. The OTA's energy consumption will rise as a result of increasing the $\mathrm{K}$ factor. 
The OTA's performance strength is mostly determined by the size of the performance transistor and the overall output power current.. The length of the transistor may be reduced to increase resistance, however this can lead to an increase in parasitic capacitance in the output nodes.

Table 1: Transistor dimensions

\begin{tabular}{|c|c|}
\hline Device & Dimensions(W/L) \\
\hline M1 & $4 / 1$ \\
\hline M2 & $4 / 1$ \\
\hline M3 & $4 / 1$ \\
\hline M Ibias & $8 / 1$ \\
\hline
\end{tabular}

\section{The Common Mode Feedback}

The common feedback circuit (CMFB) is required for full variable amplifiers in order to stabilise the amplifier's output frequency. Having a high output impedance means that the DC voltage differential between the two outputs is significantly bigger, which allows the amplifier to be eliminated and thus substantially decrease the performance of the system. The common voltage level of the amplifier appears to be determined by a common mode detector in CMFB circuits. This voltage is fed back into the amplifier in order to fix the inaccuracy in relation to the intended reference. It is possible to remedy problems by using the CMFB's topology to check all outputs with the correct reference, in this case GND.

In contrast to conventional detectors, which cover a wide range of frequencies, this approach does not require the use of wide-bandwidth resistors and capacitors. CMFB circuit loop efficiency must be maintained for all distinct pairs, which has a downside. Thus, the modulator switches' ON resistance and parasite capacitance are critical design parameters for transistors in a chopper. For a low ON resistance and a significant reduction in residual offset, a short device life is necessary. As a result, there are fewer reductions and less residual compensation when the ON resistance is minimal. In addition to being a source of noise, the resistance to switching must be minimised to a minimum.

Another crucial consideration is the way the switches work. The NMOS or PMOS option is contrasted to a CMOS option in order to eliminate the load injection that residual opposite output. However, because of the large number of computers, bacteria are often able to do better than they would otherwise. Most people will settle for an entry-level NMOS that strikes a good balance between the two constraints.

\section{Input Referred Noise}

$\mathrm{Gm}$ boosts noise reduction, but it also raises measurement or measurement inclination, or both. It is also possible to reduce noise by employing more than one $\mathrm{K}$ factor. As a result, the non-dominant pole position has higher parasites due to the increased power consumption. In order to reduce flicker noise, PMOS input devices with a slightly lower flicker coefficient are used.

\section{Designing Considerations}

For the MOS transistor the EKV model is described by the following equations $\mathrm{Ic}=\mathrm{I}_{\mathrm{D}} / \mathrm{I}_{\mathrm{S}}$

$$
\begin{aligned}
& \mathrm{gm}=\left(\mathrm{I}_{\mathrm{D}} / \mathrm{n} \phi \mathrm{t}\right) .((1-\exp (-\sqrt{\mathrm{IC}})) / \sqrt{\mathrm{I} C}) \\
& \mathrm{Is}=2 \mu \operatorname{Cox}(\mathrm{W} / \mathrm{L}) \phi^{2}
\end{aligned}
$$


Where

$\mathrm{I}_{\mathrm{D}}=$ Drain Current; $\mathrm{I}_{\mathrm{S}}=$ normalization current $; \mathrm{n}=$ slope factor $;$ Cox=oxide Capacitance; $\phi_{\mathrm{t}}^{2}$ thermal voltage at room temp; Ic= Inversion Co-efficient

If Ic $<<1$; transistor operates then in low reverse area

Ic $>1$; then transistor operates in the high reverse zone region.

\section{Results and Discussion}

\section{Critical Layout Issues}

The input chopper's parasitic must be balanced with the OTA's two input and feedback condensers, which are critical concerns. In order to minimise parasite misalignment in the chopper data, all switches' layer routings are made to be comparable. The output chopper's parasitic capacity can lead to larger cuts and residual offsets. Parasite-controlled input power is another important component of the OTA architecture. In order to ensure that the topology is as accurate as feasible, parasites must be eliminated to the greatest extent possible. The parasitic resistance is directly affected by the input capacity because of the breaks in the simulated nodes. As a result, the significance of the chopping frequency is altered.

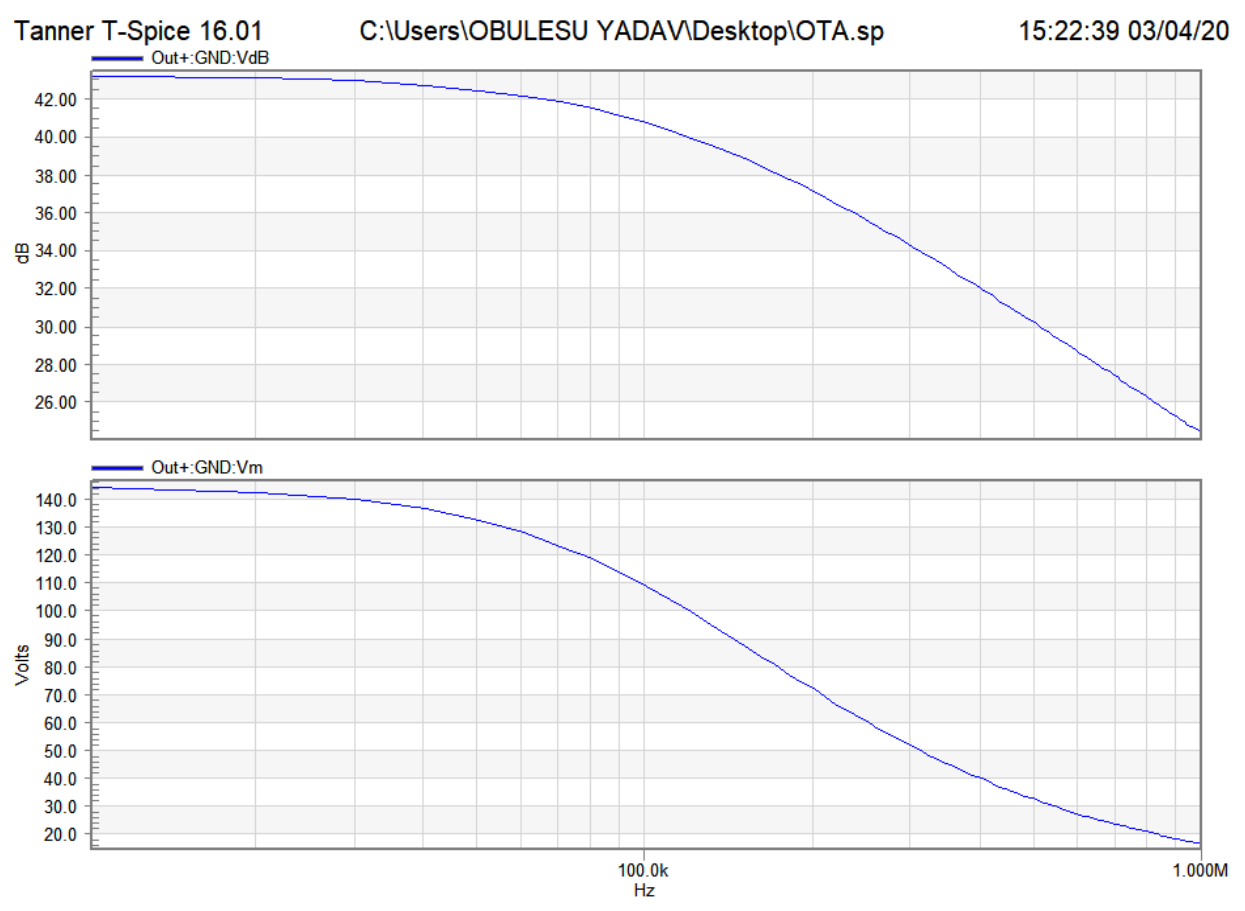

Fig 2: AC analysis of OTA

This reveals the predicted mid-band increase of $48 \mathrm{~dB}$ with the $0.06 \mathrm{~Hz}$ to $100 \mathrm{~Hz}$ frequency, the estimated ECG applications frequency range. Nevertheless, the low frequency is restricted by the parasite resistance caused by cuts. The device's frequency reaction without all the cuts shows that same early-band gain, however the small chopping frequency is $0.006 \mathrm{~Hz}$, which has been under ten years. It indicates the frequency response of the instrument amplifier without cuts. 


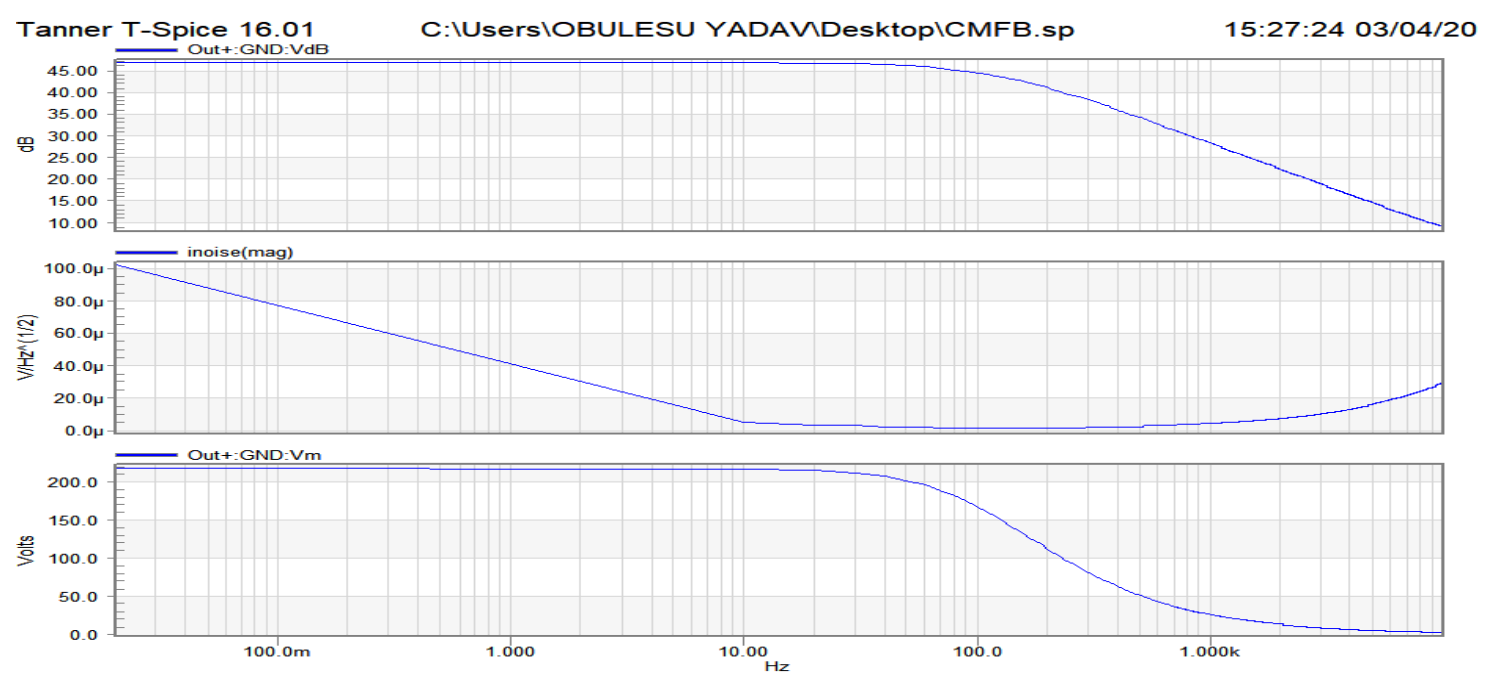

Fig 3: Bandwidth of instrumentation amplifier

The response to a $2 \mathrm{mVpp}$ sinusoidal signal at $10 \mathrm{~Hz}$ from the instrument amplifier displays a signal with a $100 \mathrm{~V} / \mathrm{V}$ gain and little noise. The machine CMRR plot values for the specified bandwidth range from 78dB to $95 \mathrm{~dB}$ and it is good enough for this submission. Good CMRR is possible with existing instrument feedback amplifiers.

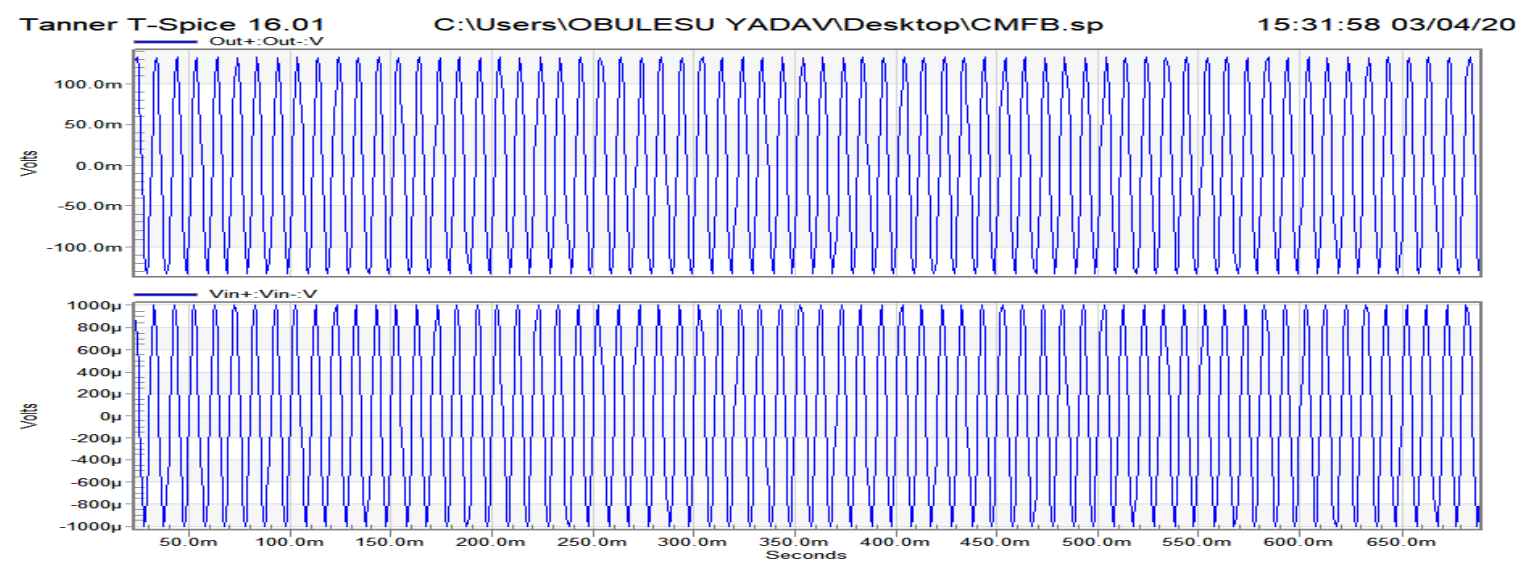

Fig 4: Transients analysis of Instrumentation Amplifier

Table 2: Output Results

\begin{tabular}{|l|l|}
\hline Parameters & Result \\
\hline Gain & $42 \mathrm{db}$ \\
\hline Bandwidth & $0.005-100 \mathrm{~Hz}$ \\
\hline CMRR & $>75 \mathrm{~dB}$ \\
\hline Input Referred Noise & $1.1 \mu \mathrm{Vrms}$ \\
\hline Power Consumption & $0.271 \mu \mathrm{W}$ \\
\hline
\end{tabular}




\section{Conclusion}

The instrument amplifier consumes $2.8 \mathrm{uW}$ of power and has a bandwidth of $0.05-100 \mathrm{~Hz}$, with a mean bandwidth gain of $40 \mathrm{~dB}$. With a flicker angle of $1 \mathrm{kHz}$, the average frequency amplitude is $1.2 \mathrm{kHz}$. As a result, the OTA virtual node cutting stage, which is parasitic resistant, limits the effectiveness of this amplification. To minimise the amplifier's impact, it is necessary to select an appropriate input power and frequency. However, achieving peak performance necessitates striking a balance between excessive noise and insufficient noise. The $0.5 \mathrm{~nm}$ CMOS ON semiconductor cycle was chosen for the instrumentation amplifier. Because of its low noise level and long service life, this amplifier could be utilised as a FE amplifier in some ECG monitoring and recording systems.

\section{References}

[1.] A. Papoulis, The Fourier Integral and Its Applications. New York: McGraw-Hill, 1962

[2.] B. Razavi, Design of Analog CMOS Integrated Circuits. New York: McGraw- Hill Science/Engineering Math,2001

[3.] C. C. Enz and G. C. Temes, "Circuit techniques for reducing the effects of op- amp imperfections: autozeroing, correlated double sampling, and chopper stabilization," IEEE Proceedings, vol. 84, pp. 1584-1614,1996

[4.] C. Yadav and S. Prasad, "Low Voltage Low Power Sub-threshold Operational Amplifier in 180nm CMOS," in 2017 Third international conference on sensing, signal processing and security (ICSSS), 2017, pp. 35-38

[5.] G. Palmisano, G. Palumbo, and S. Pennisi, "Design Procedure for Two-Stage CMOS Transconductance Operational Amplifiers: A Tutorial," Analog Integrated Circuits and Signal Processing, vol. 27, no. 3, pp. 179-189, 2001

[6.] J. F. Witte, K. A. A. Makinwa, and J. H. Huijsing, Dynamic Offset Compensated CMOS Amplifiers. Boston: Springer,2009

[7.] J. G. Webster, Medical Instrumentation: Application and Design, 4th ed. Wiley John Wiley \& Sons, INC., 2009

[8.] J. Huijsing, "Low noise and low offset operational and instrumentation amplifiers," in Operational Amplifiers, $2^{\text {nd }}$ ed., Dordrecht: Springer, 2011, pp. 355-391

[9.] J. Magri, I. Grech, O. Casha, E. Gatt, and J. Micallef, "Design of CMOS Front-End Circuitry for the Acquisition of Biopotential Signals," in 2016 IEEE International Conference on Electronics, Circuits and Systems (ICECS), 2016, pp. 161-164

[10.] K. A. Ng and P. K. Chan, "A CMOS analog front-end IC for portable EEG/ECG monitoring applications," IEEE Trans. on Circuits and Systems I: Regular Papers, , vol. 52, pp. 2335-2347,2005.

[11.] K. A. Ng and Y. P. Xu, "A Low-Power, High CMRR Neural Amplifier System Employing CMOS Inverter-Based OTAs With CMFB Through Supply Rails," IEEE Journal Of Solid-State Circuits, vol. 51, no. 3, pp. 724-737, 2016

[12.] K. D. Wise, "Silicon Microsystems for Neurosicence and Neural Prostheses," IEEE Engineering in Medicine and Biology Magazine, vol. 24, no. 5, pp. 22-29, 2005

[13.] K. Jordy, "Low-power amplifier chopper stabilization for a digital-to-analog converter," Thesis, MIT, Cambridge, MA, August2008 
[14.] K. Pratyusha, S. Kumar, and A. Kumari, "Low Power Amplifier For Biopotential Signal Acquisition System," in 2015 International Conference on Advances in Computing, Communications and Informatics (ICACCI), 2015, pp. 324-329

[15.] M. A. T. Sanduleanu and E. A. J. M van Tuijl, Power Trade-offs and Low-power in Analog CMOS ICs. Dordrecht : Kluwer Academic Publishers,2003

[16.] L. Magnelli, F. A. Amoroso, F. Crupi, G. Cappuccino, and G. Iannaccone, "Design of a 75$\mathrm{nW}$, 0.5-V subthreshold complementary metal - oxide - semiconductor operational amplifier," Internation Journal of circuit theory and applications, vol. 42, no. 9, pp. 967-977, 2014

[17.] M. Alioto, "Understanding DC Behavior of Subthreshold CMOS Logic Through Closed-Form Analysis," IEEE transaction on circuits and systems, vol. 57, no. 7, pp. 1597-1607, 2010

[18.] M. S. J. Steyaert and W. M. C. Sansen, "A micropower low-noise monolithic instrumentation amplifier for medical purposes," IEEE J. Solid-State Circuits, ,vol. 22, pp. 1163-1168,1987

[19.] S. S. Rajput, A. Singh, A. K. Chandel, and R. Chandel, "Design of Low-Power High-Gain Operational Amplifier for Bio-Medical Applications," in 2016 IEEE Computer Society Annual Symposium on VLSI, 2016, pp. 355-360.

[20.] Y. Tsividis and C. McAndrew, Operation and Modeling of the MOS Transistor, 2nd ed. Oxford university press, 2011 